\title{
Detection of small targets in a marine environment using laser radar
}

\author{
Gerard J. Kunz, Herman H.P.Th. Bekman, Koen W. Benoist, \\ Leo H. Cohen, Johan C. van den Heuvel and Frank J.M. van Putten \\ TNO Defence, Security and Safety \\ P.O. Box 96864, 2509 JG The Hague, The Netherlands
}

\begin{abstract}
Small maritime targets, e.g., periscope tubes, jet skies, swimmers and small boats, are potential threats for naval ships under many conditions, but are difficult to detect with current radar systems due to their limited radar cross section and the presence of sea clutter. On the other hand, applications of lidar systems have shown that the reflections from small targets are significantly stronger than reflections from the sea surface. As a result, dedicated lidar systems are potential tools for the detection of small maritime targets. A geometric approach is used to compare the diffuse reflection properties of cylinders and spheres with flat surfaces, which is used to estimate the maximum detectable range of such objects for a given lidar system. Experimental results using lasers operating at $1.06 \mu \mathrm{m}$ and $1.57 \mu \mathrm{m}$ confirm this theory and are discussed. Small buoys near Scheveningen harbor could be detected under adverse weather over more than $9 \mathrm{~km}$. Extrapolation of these results indicates that small targets can be detected out to ranges of approximately $20 \mathrm{~km}$.
\end{abstract}

Keywords: small targets, marine environment, laser radar, laser range finder

\section{INTRODUCTION}

Small maritime targets, like periscope tubes, jet skies, swimmers and small boats, are hot topics both for civil and navy applications, e.g., 'man overboard', illegal immigration, drugs transport and threats, e.g. the attack on the USS Cole Navy ship in Aden, Yemen, October 12, 2000. However, the detection of these targets using current radar systems is problematic due to the small radar cross-section and strong sea clutter. Stealth constructions might even further reduce radar reflections of targets. An alternative detection method was born from experiences obtained with a lidar (light detection and ranging) when measurements where carried out from the Dutch weather ship Cumulus on the Atlantic during spring 1983. Since that time other experiments, e.g., from the Meetpost Noordwijk Netherlands (in 1996 and 2000) and along the Irish west coast during the PARFORCE trials in 1999 and $2000^{1}$ confirmed this phenomena. On the other hand, other experiences learned that relatively strong signals were returned from small hard targets, e.g. poles and streetlamps, a principle that is used by laser range finders. So far, no explicit attention was paid to these effects and no quantitative information was processed. Also, knowledge on the backward reflection properties of the wavy sea surface is lacking. This was the reason to look in more detail to the detection of small maritime targets using lidar. Our current powerful lidar, operating at $1.06 \mu \mathrm{m}$, is an excellent tool to obtain detailed knowledge on the possibilities of this technique for the detection of small targets.

In this work, a geometric approach is used to calculate the signal strength returned from small diffuse reflecting cylinders and spheres using laser radar systems, which is compared with the diffuse reflection of flat diffuse reflecting surfaces. These reflections are compared with atmospheric backscatter values which are direct results of the current lidar.

Subsequently a model is developed to estimate the maximum detection range of the current lidar system for the small targets of interest. Because neither the shape, nor the reflection properties, nor the cross section of targets is known, the term 'equivalent diffuse reflection coefficient' is introduced to prevent discussions on geometry and BRDF (Bidirectional Reflectance Distribution Function) reflection properties.

The reflection of the sea surface is of utmost importance in this context, but theory is complex, difficult to handle and commonly used to predict reflections from the sun and the sky towards an observer but not to predict reflections of mono-static lidar systems or laser range finders. Therefore the choice has been made to measure these parameters with the current backscatter lidar of TNO Defense for the situations of interest.

Remote Sensing of the Coastal Oceanic Environment, edited by Robert J. Frouin, Marcel Babin, Shubha Sathyendranath, Proc. of SPIE Vol. 5885 (SPIE, Bellingham, WA, 2005) · 0277-786X/05/\$15 · doi: 10.1117/12.614914 
Within the context of this project a lidar operating at the eye-safe wavelength of $1.57 \mu \mathrm{m}$ is developed. At this wavelength the radiation is strongly absorbed in the eye and badly focused on the retina. The development of the laser and the lidar receiver is briefly discussed.

\section{Reflection of laser radiation by small targets}

The reflection properties of targets for laser radiation are, among others, a function of the geometry of that target, the spectral reflection properties, the aspect angle and the illuminated fraction of the target. This seems to be a complex problem, but using 3D segmented models of targets and the reflection properties of the individual segments it is possible to estimate the reflection for a given situation. Such a model is available from within the EOSTAR program suite ${ }^{2,3}$ developed at TNO but requires a geometric description of the target of interest. Here we limit our self to a few generic surfaces, like a flat surface, cylinder and sphere. A comparison is made between these geometries and the maximum detection range is estimated.

\section{Diffuse reflecting flat surfaces}

An equation is derived to calculate the diffusely reflected radiation from flat surfaces irradiated by a parallel beam under the assumption that the atmospheric transmission losses are negligible and that the receiver field of view is large enough to image the emitted light beam. It is also assumed that the distance to the surface is large compared to the dimensions of the surface and beam cross section. The reflection properties of the surface are expressed in the reflection coefficient $\eta$, which is equivalent to the ratio of the total reflected radiation and the total incoming radiation to the surface. By definition the reflection coefficient is equal or smaller than 1 . Also, the irradiated surface will be considered a perfect Lambertian diffuse reflector. Two different situations are considered: (1) in which the surface dimensions are large compared to the cross section of the incoming beam such as illustrated in the left panel of Figure 1 and (2) in which the surface dimensions are small compared to the incoming beam which is illustrated in the right panel of Figure 1. The first is considered as an extended target and the latter as a point target.
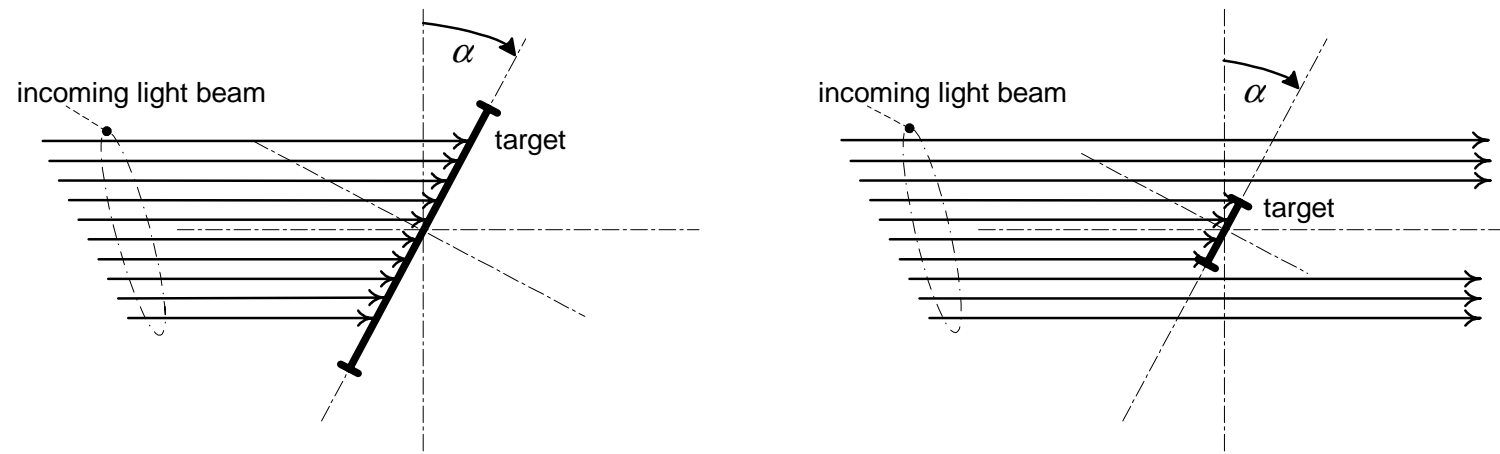

Figure 1: Left panel: an incoming light beam irradiates an extend surface under angle $\alpha$. Right panel: only a fraction of the incoming light beam irradiates a surface under angle $\alpha$, a situation that is applicable for a point target.

For the underlying study only long-distance targets are considered which are small compared to the cross section of the irradiating beam, thus situations such as shown in the right panel of Figure 1. It is assumed that a homogeneous light beam with a power of $P_{L}$ and a cross section of $A_{L}$ irradiates a point source with an area of $A_{T}$ and an equivalent diffuse reflection coefficient $\eta$. The total power that falls on this surface is:

$$
P_{0}=A_{T} \cdot \frac{P_{L}}{A_{L}} \cdot \cos (\alpha)
$$


Part of the diffusely reflected power is received by a coaxial receiver with an area of $A_{R}$ at a distance $R$ from this target which can be expressed in the form of:

$$
P_{d}(\alpha)=\frac{\eta}{\pi} \cdot A_{T} \cdot \frac{P_{L}}{A_{L}} \cdot \frac{A_{R}}{R^{2}} \cdot \cos ^{2}(\alpha)
$$

In practice, however, surfaces are rarely diffuse and the reflection coefficient depends generally on the angle of irradiation and the angle of observation, e.g., for shiny surfaces. A complete description would lead to the bidirectional reflection distribution function (BRDF), which would lead to complex equations. Moreover, these functions are rarely available. Therefore, in this work equation (2) will be used keeping in mind that this can lead to situations that the reflection coefficient is larger than 1.

\section{Diffuse reflecting cylinders}

The reflected power from small diffuse reflecting cylinders, irradiated by a parallel laser beam, is calculated under the assumptions that the diameter of the cylinder is small compared to the diameter of the incoming light beam and the detection range. The receiver is on the same axis as the incoming light beam. An impression of the applied geometry is sketched in Figure 2. The left panel gives an impression of the top view of a vertical cylinder with diameter $D$ and an incoming ray at a distance $w$ from the axis of symmetry. The right panel gives an impression of the front view of the cylinder and the width of the irradiating beam $d H$.
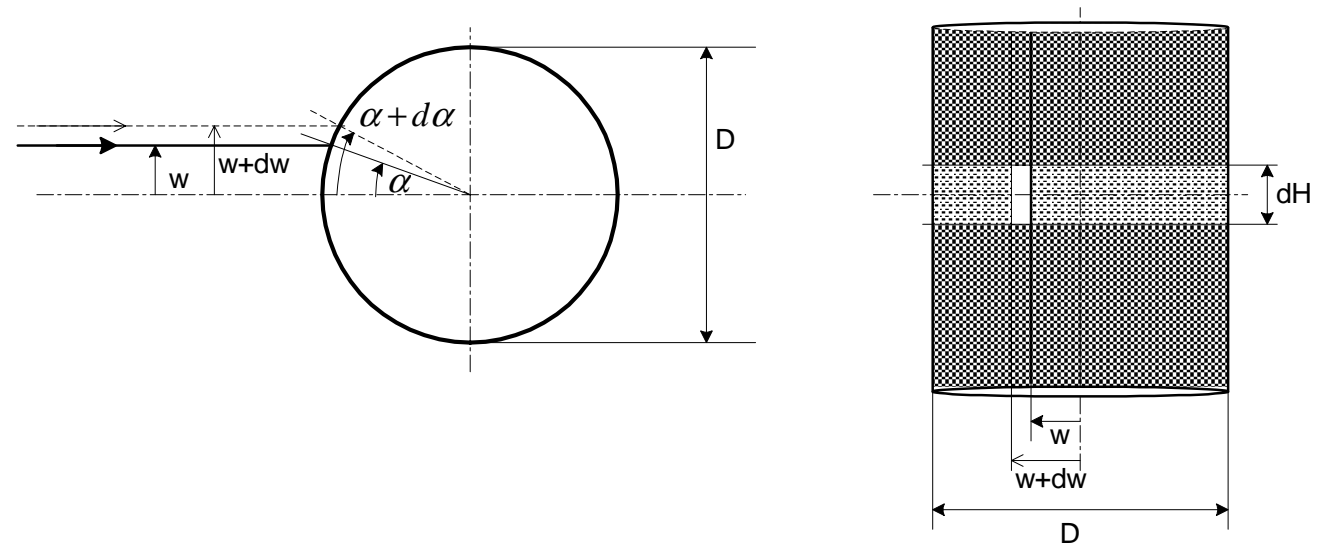

Figure 2: Left panel: top view of a vertical cylinder illuminated with a beam of light at a distance $w$ from the axis of symmetry. The right panel gives an impression of the front view with the illuminated area $d w \times d H$. Detector and source (both not sketched) are collocated at the axis of symmetry at a relatively long distance from the cylinder.

The cylinder surface is approximated by a large number of small flat fractions each at a different angle with the incoming light beam. With reference to equation (2), the signal from such a fraction at a distance $R$ from the surface under angle $\alpha$ with the incoming radiation can be written as:

$$
d P_{d}(\alpha)=\frac{\eta}{\pi} \cdot \frac{D \cdot d H}{2} \cdot \frac{P_{L}}{A_{L}} \cdot \frac{A_{R}}{R^{2}} \cdot \cos ^{2}(\alpha) \cdot d \alpha
$$

Because the cylinder is symmetrical around the vertical axis, the signal from a cylinder with height $H$ (assumed smaller than the diameter of the irradiating beam) is found by integration over the angle $\alpha$ between $-\pi / 2$ and $\pi / 2$, which results in: 


$$
P_{d}=\eta \cdot \frac{D \cdot H}{4} \cdot \frac{P_{L}}{A_{L}} \cdot \frac{A_{R}}{R^{2}}
$$

Diffuse reflecting spheres

The geometry used to calculate the reflection of diffuse reflecting spheres is sketched in Figure 3. It is assumed that the diameter of the incoming beam is much wider than the diameter of the sphere $D$ and that the beam can be represented by a large number of small rays. An incoming ray, at a distance $w$ from the axis of symmetry, is illustrated in Figure 3 .
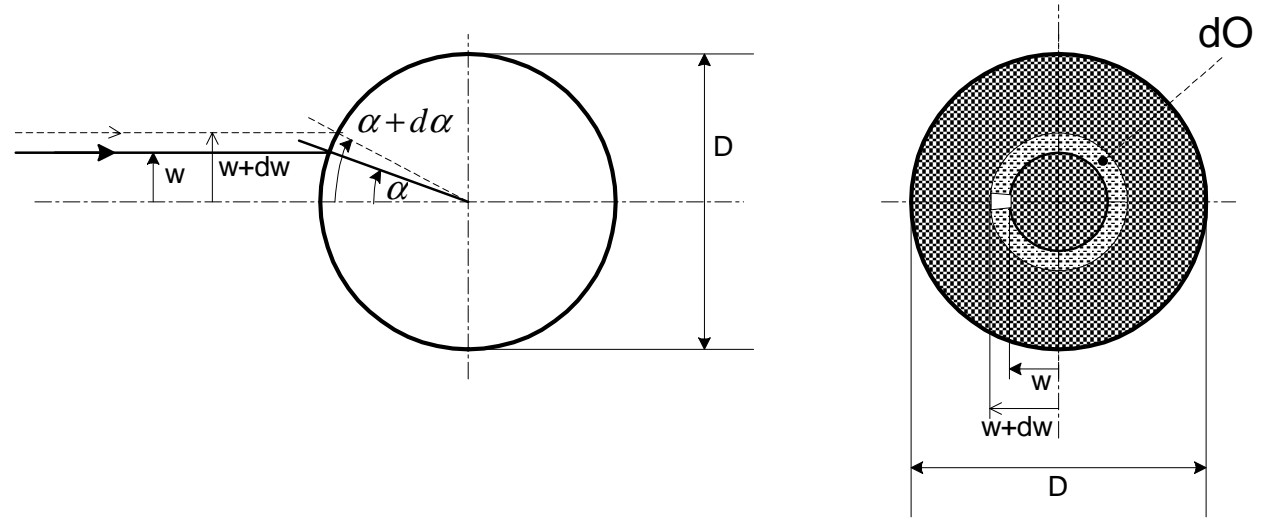

Figure 3: Left panel: top view of a sphere with diameter $D$ illuminated with a 'ray' at a distance $w$ from the axis of symmetry. Right panel: front view. The white area on the horizontal axis represents the illuminated area at a distance $w$ from the axis with width $d w$. The grey ring $d O$ represents an elementary area that is at a distance $w$ from the axis. Detector and source (both not sketched) are collocated and at a relatively long distance from the cylinder.

From a point of symmetry all the surface elements on this sphere in a ring with radius $w$ have the same angle of incidence $\alpha$ with respect to the axis of symmetry and thus contribute in a similar way to the reflected power. The area of this ring can be written as:

$$
d O=\pi \cdot D \cdot \sin (\alpha) \cdot \frac{D}{2} \cdot d \alpha
$$

With reference to equation (2), the diffuse reflection of this ring that is at an angle $\alpha$ with the incoming radiation, can be written as:

$$
d P_{d}(\alpha)=\eta \cdot \frac{D^{2}}{2} \cdot \frac{P_{L}}{A_{L}} \cdot \frac{A_{R}}{R^{2}} \cdot \sin (\alpha) \cdot \cos ^{2}(\alpha) \cdot d \alpha
$$

The total power received is found by integrating $\alpha$ between 0 and $2 \pi$ gives with area Ad at distance $\mathrm{R}$. This results in:

$$
P_{d}=\eta \cdot \frac{D^{2}}{6} \cdot \frac{P_{L}}{A_{L}} \cdot \frac{A_{R}}{R^{2}} .
$$

Discussion

Due to the curved surfaces of cylinders and spheres, these geometries will have a lower diffuse reflection than of flat surfaces with the same projected area and covered with the same material. The ratio of these reflections is found by replacing $D . H$ in equation (4) for a cylinder with $A_{T}$ and $\pi . D^{2} / 4$ in equation (7) also with $A_{T}$, which results in: 


$$
P_{F L A T}: P_{C Y L I N D E R}: P_{S P H E R E}=\frac{1}{\pi}: \frac{1}{4}: \frac{2}{3 \pi} \approx \frac{1}{3}: \frac{1}{4}: \frac{1}{5}
$$

These calculations show that spherically shaped surfaces have the lowest diffuse reflection and that cylinders with the same projected area have a $30 \%$ higher reflection. Flat surfaces, on the other hand, have a diffuse reflection which is about $60 \%$ higher than spherical surfaces with the same projected area.

Comparison of atmospheric returns with returns from hard targets

Returns from hard targets and returns from the atmosphere, both obtained with the same pulsed lidar, cannot directly be compared. The reason for this is that hard targets reflect a certain amount of energy (in a time interval equivalent to the laser pulse duration) whereas the atmosphere returns a continuous stream of power towards the receiver as a function of range and generally over much longer periods. Moreover, lidar systems express their results in backscatter coefficient (or extinction after some additional processing). One possibility to compare both types of returns is to express the returns from the hard targets $P_{H T}$ in terms of power instead of in terms of energy. This is possible by accounting for the pulse duration or by the integration time of the receiver whichever is longest. The latter is used here because the receiver integration time is generally larger (25-1000 ns and user selectable) compared to the duration of the laser pulse ( $8 \mathrm{~ns}$ ). To find the relationship between the signals from hard targets and atmospheric returns, an extended target with a diffuse reflection coefficient $\eta$ at a distance $R$ is considered which is irradiated with a laser pulse with energy $E_{L}$. The two way transmission is expressed by $T^{2}$. Using a receiver with an integration time $\tau$, the returned power can be expressed as:

$$
P_{H T}(R)=\frac{E_{L}}{\tau} \cdot \frac{1}{\pi} \cdot \frac{A_{R}}{R^{2}} \cdot \eta \cdot T^{2}
$$

On the other hand, the single scatter lidar equation for a homogeneous atmosphere can be written as ${ }^{1}$ :

$$
P_{A}(R)=E_{L} \frac{c}{2} \frac{A_{R}}{R^{2}} \beta T^{2}
$$

in which $c$ is the speed of light and $\beta$ is the atmospheric backscatter coefficient in $m^{-1} \cdot s r^{-1}$. By comparing equations (9) and (10) the diffuse reflection coefficient can be expressed in terms of the integration time and the equivalent backscatter coefficient:

$$
\eta=\frac{c \pi}{2} \tau \beta
$$

Discussion

In principle, lidar systems are developed to measure the spatial backscatter of the atmospheric aerosol and molecules. By a proper processing the returned waveforms are converted to the attenuated backscatter coefficient, which is the basis for additional inversion processes, e.g. to extinction profiles. Without knowing the origin of the signals, the lidar system cannot distinguish between returns from hard targets and atmospheric returns, thus returns from hard targets are also expressed in the same unit, but are subject to the receiver integration time. The derived conversion function given by equation (10) provides the proper conversion from backscatter in $m^{-1} . s r^{-1}$ to the dimensionless reflection coefficient.

Using the above equations it is estimated that, under a meteorological visibility of $20 \mathrm{~km}$, diffuse reflecting cylinders with a diameter of $20 \mathrm{~cm}$, a length of $1 \mathrm{~m}$ and a diffuse reflection coefficient of a few percent can easily be detected with the current lidar system ${ }^{1}$ over ranges of $10 \mathrm{~km}$ or more. Targets with a larger cross section can be detected over larger ranges. 
Specular reflections of small flat targets can be orders of magnitude larger than the diffuse reflection of the same target. The actual magnitude of the returned signal depends not only on the precise alignment of the surface with the axis of the instrument but also on the divergence of the incoming laser beam and the shape of the target. Cylindrical and spherical surfaces have a higher probability of a specular reflection and are less critical for the alignment but the magnitude of the returned signal is lower. The mathematics is beyond the scope of this paper.

Reflection and scattering at $1.06 \mu \mathrm{m}$ and $1.57 \mu \mathrm{m}$

Although the current lidar, operating at $1.06 \mu \mathrm{m}$, is an excellent tool for a feasibility study, it is not eye-safe. Therefore, an eye-safe lidar is under development operating at a wavelength of $1.57 \mu \mathrm{m}$, which radiation is absorbed in the eye and not focused on the retina. Moreover, it is also expected that the reflection properties of surfaces and the scattering properties of atmospheric particles at the eye-safe wavelength will be different. However, different references indicate that the differences are marginal. Suits ${ }^{4}, 1978$, gives a number of reflection spectra of minerals, construction materials and paintings in different wavelength bands. The graphs in this reference indicate that the reflection at $1.57 \mu \mathrm{m}$ is generally $10-50 \%$ lower than at $1.06 \mu \mathrm{m}$, depending on the material or coating. For some minerals it is the opposite. Lillesand and Kiefer $^{5}, 1973$, show spectral reflections of soil, vegetation and water. The soil reflection is about $10 \%$ higher at $1.57 \mu \mathrm{m}$ and the vegetation reflection about $20 \%$ lower than at $1.06 \mu \mathrm{m}$. Reflection spectra of different rock types between $0.4 \mu \mathrm{m}$ and $2.4 \mu \mathrm{m}$, published by Rowan, Goetz and Ashly ${ }^{6}, 1977$, show only a few percent variations between the two wavelengths of interest. Kahle ${ }^{7}, 1984$, presents more spectra with similar behavior. Numerous sites on reflection spectra can be found on the internet. A very large number of materials and painting are found on www. SpecLib. JPL. NASA. Gov1. This site shows that the reflection of metals are by a factor 2 to 3 higher at $1.57 \mu \mathrm{m}$ than at $1.06 \mu \mathrm{m}$. Paintings are $10 \%$ to $30 \%$ higher. On the other hand, asphalt has a reflection coefficient that is $30 \%$ lower at $1.57 \mu \mathrm{m}$. The reflection of seawater is somewhat lower, whereas the reflection of 'sea foam' seems to peak at about 1.57 $\mu \mathrm{m}$. Vegetation has $40 \%$ to $200 \%$ higher reflection at $1.06 \mu \mathrm{m}$ than at $1.57 \mu \mathrm{m}$.

Molecular transmission of the atmosphere calculated with Modtran showed that under normal atmospheric conditions the transmission at $1.06 \mu \mathrm{m}$ and $1.57 \mu \mathrm{m}$ is comparable. Therefore, no special molecular effects are expected. The relatively strong absorption lines at $1.1 \mu \mathrm{m}$ and $1.4 \mu \mathrm{m}$ are caused by water vapor but are outside the applied laser wavelengths.

The aerosol extinction is calculated using $\mathrm{ANAM}^{8}$, a program developed by SPAWAR and TNO for the maritime aerosol. Calculations for the spectral interval between $1.0 \mu \mathrm{m}$ and $2.0 \mu \mathrm{m}$ and a relative humidity of $83 \%$ indicate that for a wind speed of $12 \mathrm{~m} / \mathrm{s}$ the extinction coefficient at $1.57 \mu \mathrm{m}$ is approximately $10 \%$ lower that at $1.06 \mu \mathrm{m}$. Additional calculations show that this value decreases with wind speed. Therefore, it is expected that the aerosol effect is weak.

\section{Experimental results}

To verify the idea to detect small maritime targets with a laser radar system initial measurements were carried out on a relatively small vertical pole (simple antenna mast) at at a relatively short distance. This mast was used to verify the above discussed theory for both the $1.06 \mu \mathrm{m}$ and $1.57 \mu \mathrm{m}$ wavelength. Lateron, measurements were carried out on small buoys near Scheveningen harbour (North Sea coast, The Netherlands) at distances between 1 and $10 \mathrm{~km}$ with the 1.06 $\mu \mathrm{m}$ system.

Eye-safe $1.570 \mu \mathrm{m}$ laser radiation is generated using an external $\mathrm{OPO}^{9,10,11}$ which is pumped with the $1.064 \mu \mathrm{m}$ Quantel Brilliant Nd:YAG laser in the lidar. The frequency converter was constructed using two $30 \mathrm{~mm}$ long S-KTP crystals in a ring cavity with a length of $40 \mathrm{~cm}$ and an output coupler of $30 \%$. With this setup, a maximum output pulse energy of 80 $\mathrm{mJ}$ was obtained with a beam quality of $M^{2}=6.1$ using a pump energy of $300 \mathrm{~mJ}$. However, the system was operated at an output of $30 \mathrm{~mJ}$ to prevent damage to the crystals. A long-wave pass filter in combination with a silicon window was used to suppress the residual $1.06 \mu \mathrm{m}$ radiation. The receiver for the eye-safe lidar was built around a $0.2 \mathrm{~mm}$ diameter

\footnotetext{
${ }^{1}$ Reproduced from the ASTER Spectral Library through the courtesy of the Jet Propulsion, Laboratory, California Institute of Technology, Pasadena, California. Copyright, 1999, California Institute of Technology.
} 
InGaAs avalance photo diode with a temperature controlled bias voltage and a Celestron 750 telescope. A small aspherical lens was used to focus the collected radiation on the detector. The received radiation was converted to an electrical signal using a transimpedance amplifier and a $50 \Omega$ line driver. The transfer of this combination is $210 \mathrm{kV} / \mathrm{W}$ with an equivalent noise power of $8.5 \mathrm{nW}$ and a signal bandwidth of $20 \mathrm{MHz}$.

Antenna mast at $1.06 \mu \mathrm{m}$ and $1.57 \mu \mathrm{m}$

Introductory lidar measurements were carried out from the roof of the laboratory at a height of about $10 \mathrm{~m}$ above the ground from which we had a view on a small vertical antenna mast on the top of a high rise at a distance of about $550 \mathrm{~m}$. The dimensions of the mast were estimated from a photograph under the assumption that the heights of the levels in the building are $3 \mathrm{~m}$. From this photograph we guess that the mast diameter is about $15 \mathrm{~cm}$ and the height about $8 \mathrm{~m}$.

Because the divergence of the laser beam is on the order of $1 \mathrm{mrad}$ only a fraction of the beam illuminates the mast. The lidar system was operated at a significantly reduced receiver diameter and laser enegy. The receiver integration time was increased to $100 \mathrm{~ns}$ using a first order low pass filter. To be sure that at least a few returns from the mast were obtained, the $20 \mathrm{~Hz}$ system was scanned in a small horizontal interval (PPI = plan position indicator) of 9.5 degrees at a low speed of 2.5 degrees per second. This scan provided 495 lidar waveforms from which 6 were completely over the mast. The mast reflections showed to be in good contrast with the atmospheric returns equivalent to a backscatter coefficient of 6.3 $10^{-5} \mathrm{~m}^{-1} \cdot \mathrm{sr}^{-1}$ (comparable to the backscatter of a very dense cloud). Using equation (11) this backscatter is equivalent to a diffuse reflection coefficient of $0.3 \%$, under the assumption of an extended target. However, this is not the case because the angular size of the mast $(15 \mathrm{~cm} / 550 \mathrm{~m}=0.27 \mathrm{mrad})$ is about an order of magnitude smaller than the angular size of the beam $(9.5 * 6 / 495=0.12 \mathrm{deg}=2.1 \mathrm{mrad})$. Therefore, it is estimated that the diffuse reflection coefficient of the mast surface is about a factor $8(2.1 / 0.27)$ larger thus about $2.5 \%$. Repeated measurements with the eye-safe system showed that the diffuse reflection at $1.57 \mu \mathrm{m}$ is about $1 \%$. By assuming that the mast is a cylinder, the actual diffuse reflection is about $30 \%$ higher.

Maritime surface targets and waves

A suitable location with small-unmanned maritime targets was searched along the Dutch coast, to show the principle of detecting small targets at the sea surface. The location of interest should have some small objects in the water, preferably at least several kilometers from the observation point, and easily accessible for man and equipment. The best location appeared to be the southern Scheveningen harbour pier with a length of about $600 \mathrm{~m}$ at approximately $\left(52^{\circ} 6^{\prime} \mathrm{N} ; 4^{\circ} 15^{\prime} \mathrm{E}\right)$. From this position several buoys at distances between $1 \mathrm{~km}$ and $10 \mathrm{~km}$ are visible, and even buoys at larger distances near the Rottterdam harbour entrance.
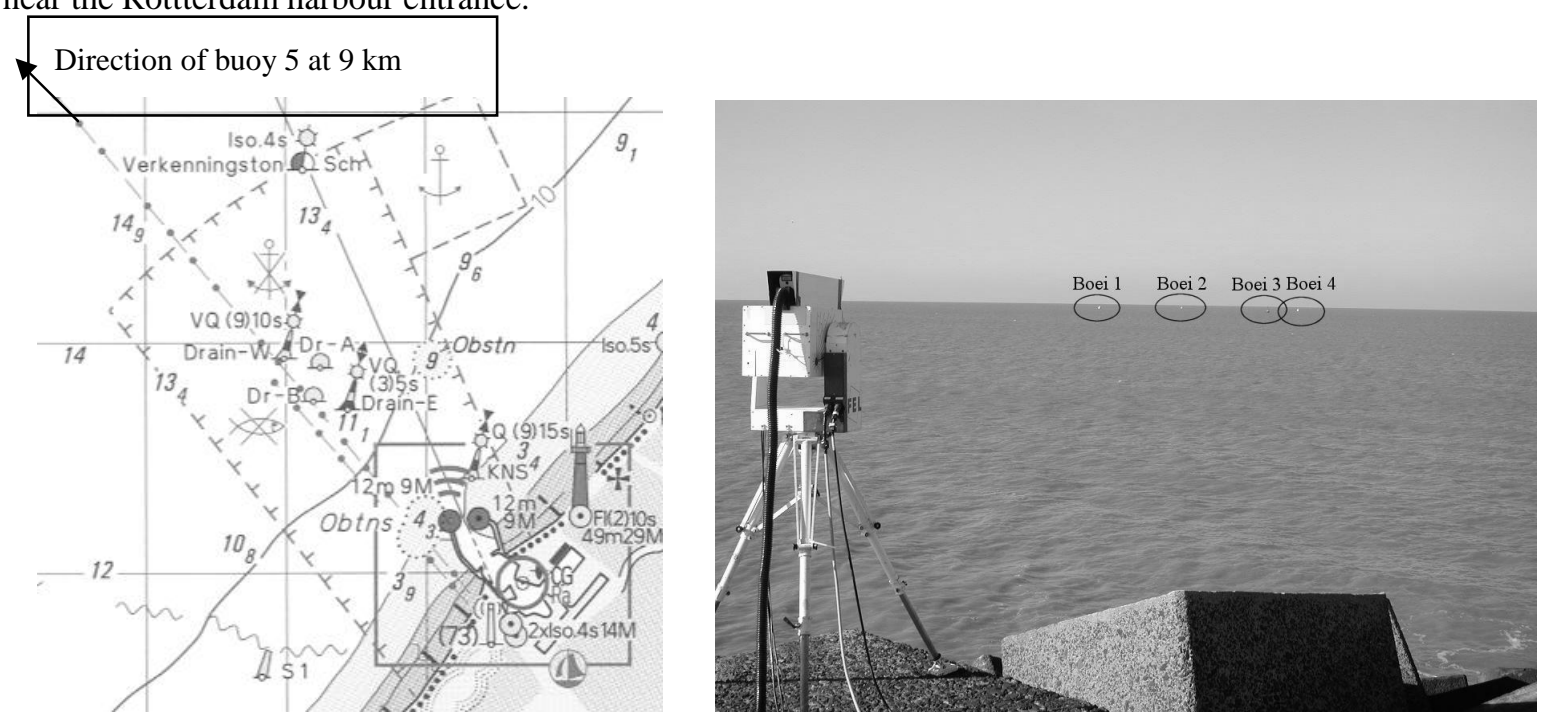

Figure 4: Left panel: Magnified portion of Hydrographic Map 1801.7, Royal Netherlands Navy, 'Dienst der Hydrography', near the Scheveningen harbour entrance. The lidar was positioned at the end of the southern pier, near the green traffic light (near text 'Obtns'). The four buoys of interest (from left to right) are: DR-B (Boei 1), Drain-W (Boei 2), Dr-A (Boei 3) and Drain-E (Boei 4). The rectangles in the background of the map are approximately $1 \times 2 \mathrm{~km}^{2}$. Right panel: The four yellow buoys are: Boei1 is DR-B at 1.4 $\mathrm{km}$, Boei 2 is Drain-W at $1.9 \mathrm{~km}$, Boei 3 is Dr-A at $1.2 \mathrm{~km}$ and Boei 4 is Drain-E at $1.6 \mathrm{~km}$. The lidar, mounted on the tripod, is visible in the front. 
During working days shipping traffic is very limited near Scheveningen. A magnified portion of the hydrographic map, shown in Figure 4, gives an impression of the location including the identifications and positions of the buoys. A view over the sea in NW direction from the southern harbour pier, with the lidar in the front and the buoys in the back is shown in the right panel. The equipment was set up at the end of the southern Scheveningen harbour pier, about $500 \mathrm{~m}$ from the surf zone and the measurements were carried out in north-westerly directions towards open sea. No shipping trafic was observed during the experiments. The weather conditions were good. Cloudless sky, wind speed 6-7 m/s, wind direction 70 degrees, air temperature $12{ }^{\circ} \mathrm{C}$ and air pressure $1031 \mathrm{hPa}$. The water temperature was not specified in the weather report, but based on previous information in this time of the year it is assumed that the water temperature is about $16^{\circ} \mathrm{C}$. The wave height was about $0.5 \mathrm{~m}$ and whitecaps were only occasionally observed.

The four yellow buoys indicated in the left panel of Figure 4 were the objects of interest during this experiment. Two other nearby buoys are available but not measured. The very small one in south-westerly direction at a distance of about $2 \mathrm{~km}$, indicated with S1 in Figure 4, could also easily be detected. It is remarkable that the distance to this very small buoy, estimated by the human eye, seems to be much larger. On the other hand, the large buoy in north-westerly direction at a distance of about $3 \mathrm{~km}$, indicated with Iso.4S, 'Verkenningston', seems to be at a much smaller distance. Because the latter was so large and evidently visible by eye, no explicit measurements were carried out to this buoy.

Experiments were carried out in different modes: (1) by scanning the lidar in vertical direction, the so called 'range to height indicator' or RHI mode, (2) by scanning the lidar in horizontal direction, the so called 'plane position indicator' or PPI mode and (3) by measuring in a fixed direction. All the measurements were carried out at a reduced laser pulse energy of $20 \mathrm{~mJ}$.

The results of a RHI scan over Buoy 4, presented in Figure 5, show the atmospheric backscatter coefficient (coded in false colour) in a vertical plane. The lidar is in the most left position of the coloured segment. Range is on the horizontal axis $(500 \mathrm{~m} / \mathrm{div})$ and height is on the vertical axis $(100 \mathrm{~m} / \mathrm{div})$. The grey area above the first horizontal grid line represents atmospheric returns whereas the dark area below this line represents returns from the sea. The latter area is darker, indicating a low level return signal. The strong return at a distance of about $1600 \mathrm{~m}$ (see arrow) is caused by the laser light reflected from buoy Drain-E (Boei 4) at a distance of 1594 m (see Figure 4).
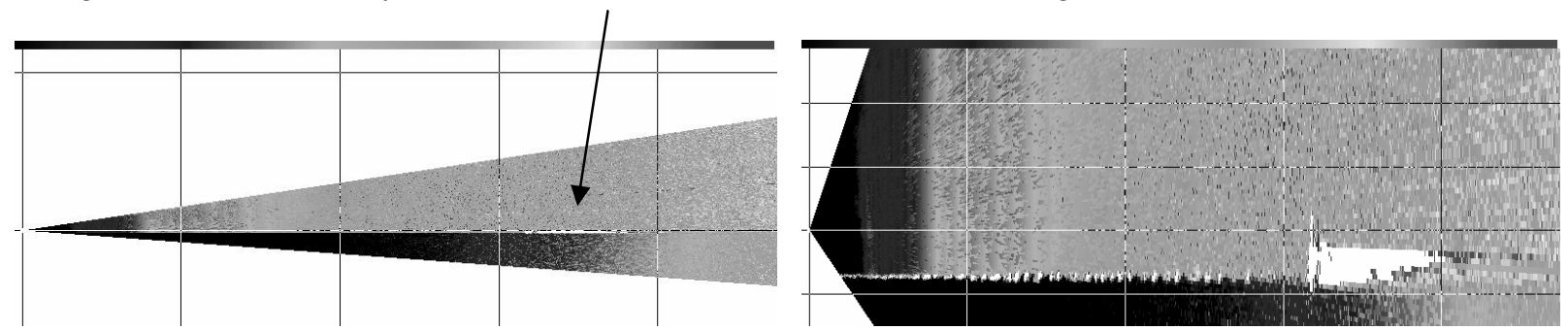

Figure 5: Left panel: Vertical scan over Buoy \#4 constructed from 512 lidar waveforms recorded in 25 seconds. The arrow indicates the buoy position. The horizontal scale is $500 \mathrm{~m} / \mathrm{div}$ and the vertical scale $100 \mathrm{~m} / \mathrm{div}$. The grey scale ranges from -7 to $-6 \log \left(\mathrm{m}^{-1} . \mathrm{sr}^{-1}\right)$. Data measured October 15, 2003, 12:18 LT (file 15121813.ldt). Right panel: Same data left panel, but re-plotted over a smaller vertical interval with $10 \mathrm{~m} /$ div with the same horizontal scale. Note that the buoy at about $1600 \mathrm{~m}$ was detected in approximately 10 individual lidar waveforms.

To better visualize the air-sea boundary and the reflections from buoy the vertical axis is stretched and the data reprocessed for this scale in the right panel of Figure 5. Although the information in both panels is based on the same dataset the stretched data show more interesting features. First, the boundary between the atmopsphere and the sea surface is more pronounced. The lidar is in the left corner point of the coloured segment and therefore the height of the lidar above the water is apparently about $7.5 \mathrm{~m}$. The large white area at a distance of approximately $1600 \mathrm{~m}$ represents the reflection from the buoy, which extends to about $5 \mathrm{~m}$ above the sea surface. The 'length' of the reflection in the figure is about 400 $\mathrm{m}$, which is an indication that the returned signal from the buoy saturated the lidar receiver, despite the reduced laser energy and the applied electronic attenuation of a factor 10. It is recommend in future experiments to further attenuate the lidar sensitivity to suppress atmospheric returns and returns from the sea surface. Remarkable are the small white spots at a regular spacing of approximately $25 \mathrm{~m}$ at the surface, which are caused by reflections from the wavy surface but the returned power and energy of these waves is much less than the return from the buoy. Other features of the wavy surface are visible in Figures 6 and 7. Surface reflections between the waves are not present. 
Although measurements in a vertical plane provide information on the height of targets, horizontal measurements (PPI mode) are more suited for searching purposes over a wider area. Horizontal lidar measurements were carried out at 10 different elevation angles from $+0.46^{\circ}$ degrees down to $-0.49^{\circ}$ to observe buoy reflections at different heights. Results obtained from one of these measurement under the elevation angle of $-0.24^{\circ}$ are presented in Figure 6 . The lidar is in the right most position in this figure.

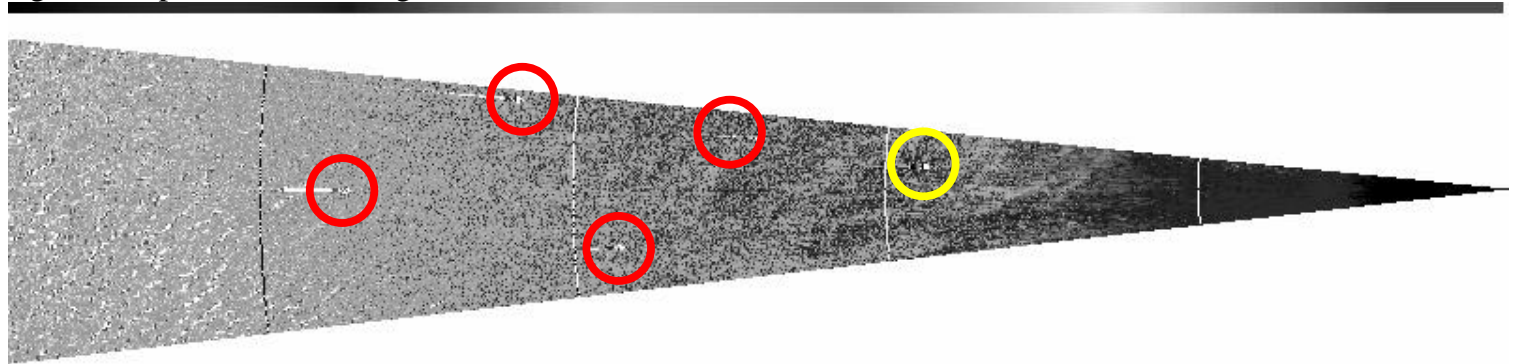

Figure 6: Results of a horizontal lidar scan over an azimuth angle interval of 12 degrees and at an elevation angle of $-0.24{ }^{\circ}(219$ waveforms). Reflections from the buoys are indicated with circles. The upper right circle is not a buoy but probably the reflection from a sea gull. Distances between the concentric range indicators are $500 \mathrm{~m}$. Data measured October 15, 2003, 13:32:45 LT.

The circles in Figure 6 indicate the positions of the buoys in the water whereas the strong reflection encircled by the upper right circle is probably from a flying sea gull, which was at a different location in the subsequent scan. The regular fringes at ranges larger than $2 \mathrm{~km}$ from the lidar (in the most left part in the figure) are reflections from the wave tops, illuminated by the finite laser beam. The distances between the lidar position and the buoys are respectively $1431 \mathrm{~m}$, $1869 \mathrm{~m}, 1206 \mathrm{~m}$ and $1593 \mathrm{~m}$, which were inverted from the lidar data recorded with a range resolution of $3 \mathrm{~m}$. These distances were in excellent agreement with the values obtained with a laser range finder of respectively $1428 \mathrm{~m}, 1868 \mathrm{~m}$, $1208 \mathrm{~m}$ and $1594 \mathrm{~m}$. Actually, the data-density in Figure 6 is too high to emphasize the reflections from the small surface targets. Therefore, the data are first plotted at a higher spatial resolution to present more detail in the data. Subsequently the data is plotted using a different color scale. For example, the left panel of Figure 7 shows the same data as presented in Figure 6 but projected over a grid of $100 \times 100$ m. The right panel in Figure 7 shows the data at a somewhat lower elevation angle, recorded 13 seconds later (note that the ghost reflection moved).
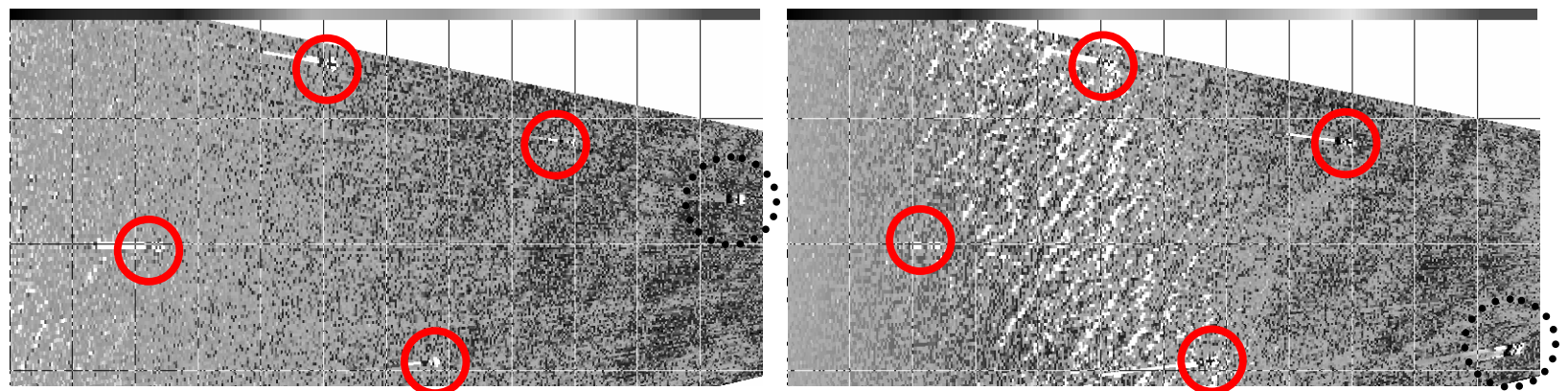

Figure 7: Left panel: same data as presented in Figure 6, but projected over a smaller area with a grid spacing of $100 \mathrm{x} 100 \mathrm{~m}$. The four circles represent the four buoys in front of the dock entrance. Right panel: data recorded at an elevation angle of $-0.31{ }^{\circ}$ but 13 seconds later. The pattern in the middle of this figure are caused by reflections from the wavy surface.

The results in Figure 7 show that the lengths of the target reflections (duration) increase with distance, thus reflections from buoys at larger ranges have a stronger overload effect on the receiver than reflectons from nearby buoys. Probably only the top fraction of nearby buoys is illuminated. For larger ranges, the beam is closer to the surface and has a wider cross-section, which results in a better illumination of the buoys, and thus in a stronger reflection. Note that the length of the reflection is more than $100 \mathrm{~m}$, which is not realistic, but caused by saturation of the receiver. In theory, the duration of the return should be on the order of the laser pulse width, thus no more than $6 \mathrm{~m}$. Detailed analysis indicate that the buoy reflections are about a factor 100 stronger than the atmospheric returns, which emphasize again the dominance of laser reflections by hard targets. In the righ panel the surface wave reflections are at shorter ranges than those in the left panel because the latter was measured at a higher elevation angle. 
So far, the data has been processed to visualize as much information as possible, thus over a wide dynamic range of signal amplitudes. As a result of this choice also the reflections of the atmosphere and the waves are clearly visible and the reflections from the buoys seemed to be embedded in the clutter. However, the signals from the buoys are several orders of magnitude stronger than the background thus by a proper selection of the color interval only the returns from the buoys are emphasized. Figure 8 shows the same data as presented in the right panel of Figure 7, but using an amplitude interval that is two orders of magnitude less sensitive.

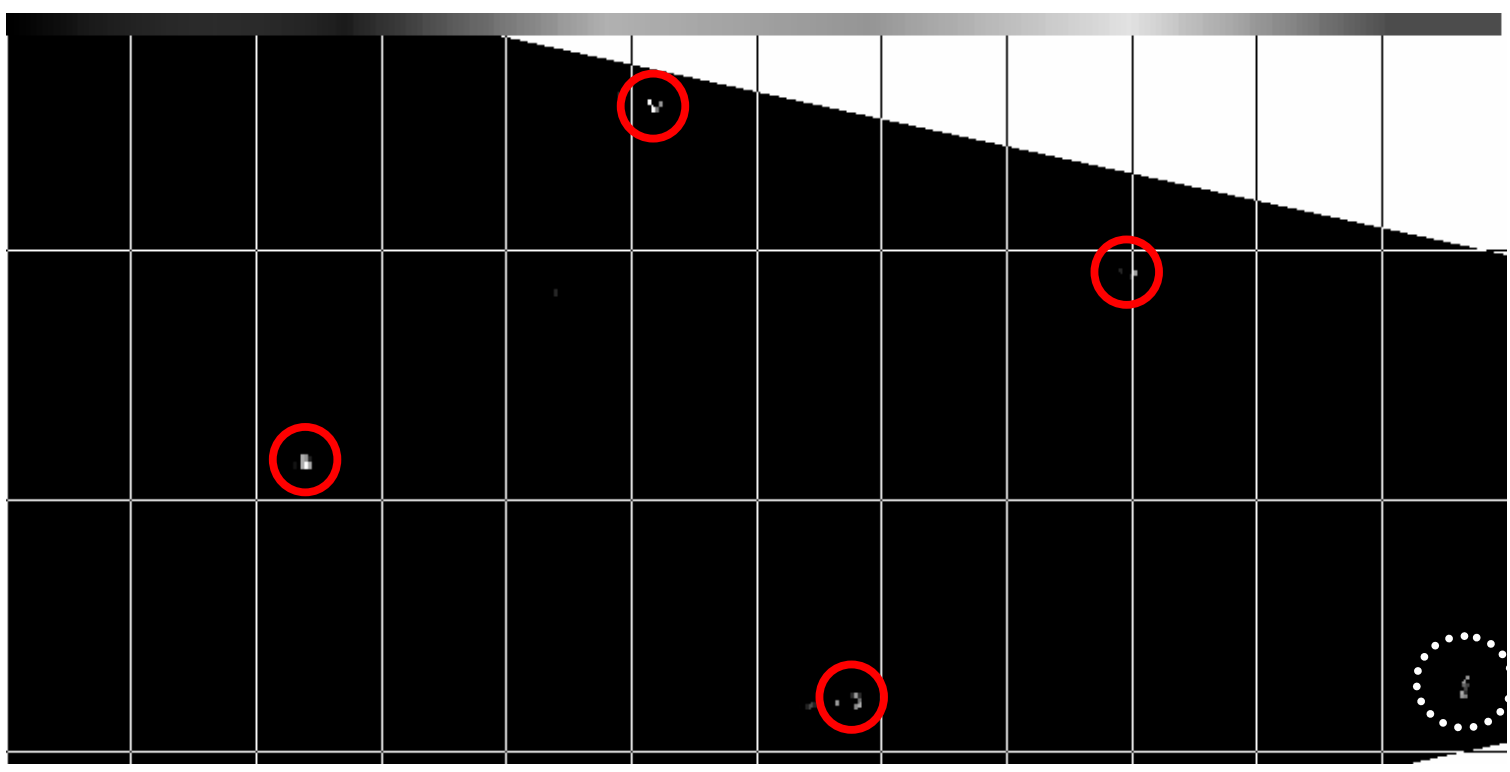

Figure 8: Same data as presented in the right panel of Figure 7 but coded in a color scale that is a factor 100 less sensitive. Due to this selection not only the atmospheric return is suppressed but also the reflections from the surface waves. Only the buoy reflection (and of the gull) remain.

The reflections of the buoys are several orders of magnitude stronger than the reflections from the atmospheric or from the waves. Of course, this will depends on the range $(R)$ because the strength of the atmospheric returns decreases with at least $R^{2}$ whereas the returns from point targets decrease with $R^{4}$.

During later experiments, the lidar could successfully measure the position and reflections of the spar buoy ' $\mathrm{S} 1$ ' in southwesterly direction at a distance of about $2 \mathrm{~km}$, from the spherical buoy 'Iso.4S, from the Verkenningston Sch' in northwesterly direction at a distance of about $3 \mathrm{~km}$ and also from the conical buoy 'F1.Y.5s, Houtrust' in northwesterly direction at a distance of $9.3 \mathrm{~km}$. The latter could not be observed with the naked eye under these prevailing conditions and also not with the video system in the lidar, but only slightly using a binocular. Because the angular resolution of the lidar was not high enough for automatic alignment or scanning, the lidar was aimed manually to the buoy. Nevertheless, the buoy could be detected with a signal-to-noise ratio of about 140. A screen snapshot of the waveform recorder is shown in Figure 9. The lidar waveform presented in Figure 9 is recorded with a time base of $10 \mu \mathrm{s} / \mathrm{div}$ and a pretrigger of $10 \mu \mathrm{s}$. The gradual decrease in the signal between about $10 \mu \mathrm{s}$ and $60 \mu$ s represents the atmospheric backscatter, attenuated by the geometric effect and the atmospheric transmission losses. Due to the application of an analog logarithmic amplifier, which suppresses large signal amplitudes and enhances low signal amplitudes, the large dynamic range of the signal could be covered within a single waveform. The relatively strong peak, visible at $62 \mu \mathrm{s}$ after the pretrigger, comes from the buoy at $9.3 \mathrm{~km}$ from the lidar. Inversion of this signal shows that the signal-to-noise ratio of this peak is a factor 140 . 


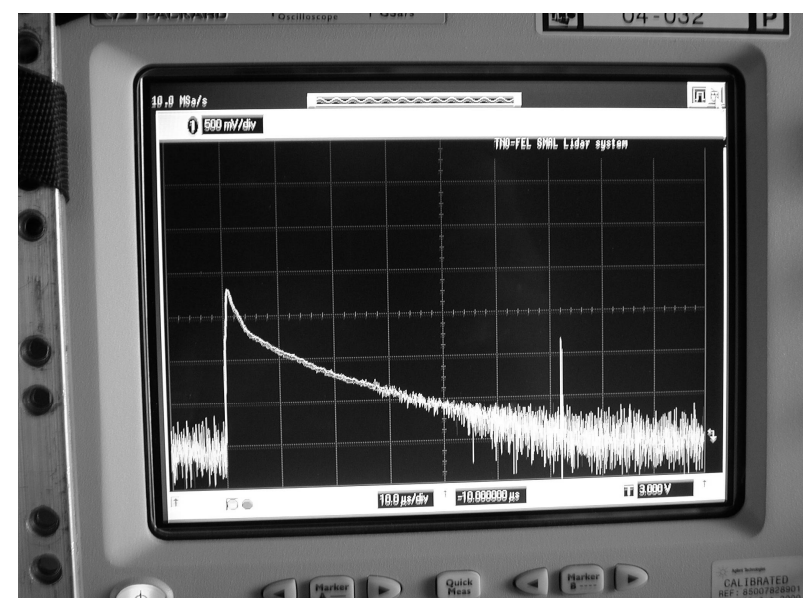

Figure 9: Photo of the oscilloscope screen with a lidar waveform at $1.064 \mu \mathrm{m}$ over a total time interval of $90 \mu \mathrm{s}$ and a pretrigger of 10 $\mu \mathrm{s}$. The laser pulse is emitted at $\mathrm{t}=10 \mu \mathrm{s}$ and the sharp signal peak at $\mathrm{t}=72 \mu \mathrm{s}$ is the reflected signal from buoy 'El.Y $5 \mathrm{~S}$, Houtrust' a distance of $9.3 \mathrm{~km}$ (reflected laser pulse round trip time is $62 \mu \mathrm{s}$ ). The analog lidar signal is converted using a logarithmic amplifier, which suppresses the strong signals and enhances the weak signal. Consequently, the noise seems to be very dominating.

\section{Conclusions}

Lidar measurements in maritime environments, e.g., lidar measurements on the Irish coast and from the Meetpost Noordwijk, showed very low (or no) reflections from the sea surface whereas strong reflections of (small) targets were measured over tens of kilometers. A combination of these two phenomena led to the conclusion that it would be possible to detect small targets on the sea surface, like buoys, periscopes, jet skies, swimmers and speed boats. The results described in this paper focused on this subject. First a model has been developed to estimate the signal strength from some geometric objects. This model shows that using the current $1.06 \mu \mathrm{m}$ lidar (energy $0.2 \mathrm{~J}$, pulse duration $10 \mathrm{~ns}$ and receiver cross section $0.02 \mathrm{~m}^{2}$ ) it is possible to detect a $15 \mathrm{~cm}$ diameter dark cylinder with a diffuse reflection coefficient of $3 \%$ over ranges of $10 \mathrm{~km}$ or more, depending on the conditions. This theory has first been verified by measurements on a $6 \mathrm{~cm}$ diameter antenna mast at a distance of $600 \mathrm{~m}$ and lateron by measurements on small buoys near Scheveningen harbour out to distances of more than $9 \mathrm{~km}$. Reflections from the antenna mast at $1.57 \mu \mathrm{m}$ are slightly lower than at 1.06 $\mu \mathrm{m}$. Lidar measurements over the sea during near gale conditions show that parts of non breaking waves at about $2 \mathrm{~km}$ from the coast have an equivalent diffuse reflection coefficient that varies between $1.10^{-5}$ and $1.10^{-4}$, which is lower than the equivalent diffuse reflection of the antenna mast. Moreover, reflections from hard targets have a larger vertical extent. The target-to-background ratio can be improved by using shorter laser pulses in combination with a wide band receiver and lasers with a smaller beam divergence. Surface reflections between the waves have never been observered with the current lidar, probably due to the strong specular forward reflection and the strong absorption of the radiation by the water. The best guess is probably that the reflection of the water surface is lower than the detection limit of the lidar, which is better than $1.10^{-6}$ in terms of equivalent diffuse reflection coefficient of an extended target.

\section{References}

1. G.J. Kunz, G. de Leeuw, E. Becker and C.D. O'Dowd, 'Lidar observations of atmospheric boundary layer structure and sea spray aerosol plumes generation and transport at Mace Head, Ireland (PARFORCE experiment), Journal of Geophysical Research 107(D19), 11.1-11.14 (2002).

2. G.J. Kunz, M.M. Moerman, A.M.J. van Eijk, S.M. Doss-Hammel and D. Tsintikidis, 'EOSTAR: an electro-optical sensor performance model for predicting atmospheric refraction, turbulence, and transmission in the marine surface layer,' in Optics in atmospheric propagation and adaptive systems VI, J.D. Gonglewski and K. Stein, Eds., Proc. SPIE Vol. 5237, pp. 81-92, Bellingham, Washington, USA (2003).

3. G.J. Kunz, M.A.C. Degache, M.M. Moerman, A.M.J. van Eijk, F.P. Neele, S.M. Doss-Hammel and D. Tsintikidis, 'Status and developments in EOSTAR, a model to predict IR sensor performace in the marine environment,' in 
Optics in atmospheric propagation and adaptive systems VII, J.D. Gonglewski and K. Stein, Eds., Proc. SPIE Vol. 5572-12, pp. 1-12, Bellingham, Washington, USA (2004).

4. G.H. Suits, Natural sources, Chapter 3 in W.L.Wolfe and G.J. Zissis, Eds., The infrared handbook $1978,1978$.

5. T.M. Lillesand and R.P. Kiefer, Remote sensing and image interpretation, New York: John Wiley \& Sons, 1973.

6. L.C. Rowan, A.F.H. Goetz and R.P. Ashley, 'Discrimination of hydro thermally altered and unaltered rocks in visible and near infrared multispectral images,' Geophysics 42(3), 522-535, 1977.

7. A.B. Kahle, Measuring spectra of arid lands, in Deserts and arid lands, El-Baz. F, Eds., pp. 195-217, Martinus Nijhoff Publishers, The Hague, The Netherlands, 1984.

8. S.G. Gathman, A.M.J. van Eijk and L.H. Cohen, 'Characterizing large aerosols in the lowest level of the marine atmosphere,' in Propagation and Imaging through the Atmosphere II, L.R. Bissonnette, Eds., Proc. SPIE Vol. 3433, pp. 41-52, Bellingham, Washington, USA (1998).

9. R.L. Burnham, J.L. Kasinski, L.R. Marshall, Eye-safe laser systems, US Patent No. 5181211, 1993.

10. G.A. Rines, D.G. Rines, P.F. Moulton, Efficient, High-Energy, KTP Optical Parametric Oscillators Pumped with 1 $\mu \mathrm{m}$ Nd-Lasers, OSA Proceedings on Advanced Solid State Lasers, Washington DC, Vol. 20, pp. 461-463, 1994.

11. B. Boulanger, M.M. Fejer, Study of gray-tracking at 1064, 532, and 355 nm, Applied Physics Letters 65 (19) 1994. 